\title{
IMP-ICDX: an injury mortality prediction based on ICD-10-CM codes
}

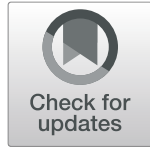

Muding Wang ${ }^{1 *}$ D, Wusi Qiu², Yunji Zeng ${ }^{3}$, Wenhui Fan' ${ }^{1}$ Xiao $\operatorname{Lian}^{3}$ and Yi Shen ${ }^{4}$

\begin{abstract}
Background: The International Classification of Diseases, Ninth Edition, Clinical Modification (ICD-9-CM) Injury Severity Score (ICISS) is a risk adjustment model when injuries are recorded using ICD-9-CM coding. The trauma mortality prediction model (TMPM-ICD9) provides better calibration and discrimination compared with ICISS and injury severity score (ISS). Though TMPM-ICD9 is statistically rigorous, it is not precise enough mathematically and has the tendency to overestimate injury severity. The purpose of this study is to develop a new ICD-10-CM injury model which estimates injury severities for every injury in the ICD-10-CM lexicon by a combination of rigorous statistical probit models and mathematical properties and improves the prediction accuracy.

Methods: We developed an injury mortality prediction (IMP-ICDX) using data of 794,098 patients admitted to 738 hospitals in the National Trauma Data Bank from 2015 to 2016. Empiric measures of severity for each of the trauma ICD-10-CM codes were estimated using a weighted median death probability (WMDP) measurement and then used as the basis for IMP-ICDX. ISS (version 2005) and the single worst injury (SWI) model were re-estimated. The performance of each of these models was compared by using the area under the receiver operating characteristic (AUC), the Hosmer-Lemeshow (HL) statistic, and the Akaike information criterion statistic.

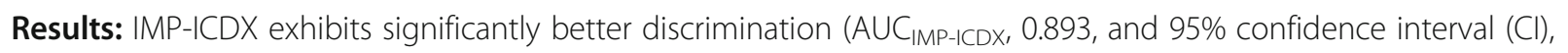
0.887 to 0.898 ; $A \cup C_{\text {ISS, }} 0.853$, and $95 \% \mathrm{Cl}, 0.846$ to 0.860 ; and $A \cup C_{S W l}, 0.886$, and $95 \% \mathrm{Cl}, 0.881$ to 0.892 ) and

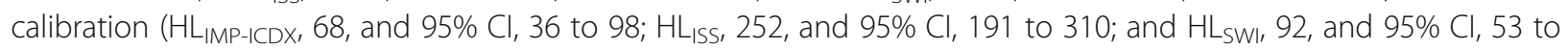
128) compared with ISS and SWI. All models were improved after the extension of age, gender, and injury mechanism, but the augmented IMP-ICDX still dominated ISS and SWI by every performance.
\end{abstract}

Conclusions: The IMP-ICDX has a better discrimination and calibration compared to ISS. Therefore, we believe that IMP-ICDX could be a new viable trauma research assessment method.

Keywords: International Classification of Diseases Tenth Edition (ICD-10-CM), Injury mortality prediction for ICD-10-CM (IMP-ICDX), Injury severity score (ISS), Mortality prediction

\section{Introduction}

Trauma score methods can be divided into two categories of systems. First, the injury severity score (ISS), the new injury severity score (NISS), the tangent injury severity score (TISS), the trauma mortality prediction model (TMPM), and injury mortality prediction (IMP) [1-5] score methods based on the Abbreviated Injury Scale (AIS) [6] lexicon. Their ability of predicting trauma death is also improved $[2-5,7]$.

\footnotetext{
* Correspondence: hzwangmd@hotmail.com

${ }^{1}$ Department of Emergency Medicine, Affiliated Hospital of Hangzhou Normal University, 126 Wenzhou Road, Gongshu District, Hangzhou 310015, Zhejiang, People's Republic of China

Full list of author information is available at the end of the article
}

However, the AIS codes must be evaluated by trauma surgeon experts. In these circumstances, a great deal of manpower and material resources is consumed. It is difficult for developed countries, let alone developing ones. These situations hinder the trauma score in-depth research and popularization. Second, the International Classification of Diseases Ninth Edition (ICD-9-CM) Injury Severity Score (ICISS) and the trauma mortality prediction model (TMPM)-ICD9 score methods based on ICD-9-CM lexicon [8, 9]. ICD-9-CM codes are the common disease diagnosis codes around the world. Currently, most countries and regions apply the updated ICD-10-CM. The number of diagnostic categories available is approximately

(c) The Author(s). 2019 Open Access This article is distributed under the terms of the Creative Commons Attribution 4.0 International License (http://creativecommons.org/licenses/by/4.0/), which permits unrestricted use, distribution, and 
over 9000, which is more than the number of AIS code categories. Although ICD-10-CM codes are not similar to AIS which implies injury severities, each diagnosis has implied the information of anatomy trauma, a variety of disease severity, and the possibility of mortality. ICD-10-CM codes also include the possibility of death, such as traumatic hemorrhage of right cerebrum with loss of consciousness of $30 \mathrm{~min}$ or less, initial encounter; displaced fracture of base of neck of right femur, initial encounter for closed fracture; and major laceration of liver, initial encounter.

The ICISS is the product of empirically derived survival risk ratios (SRRs) for trauma ICD-9-CM codes [8]. SRR is a survival rate of all trauma patients in a specific trauma ICD-9-CM code. It contains survival rates of patients who sustained both a single injury and multiple injuries. Although ICISS is better than the ISS and NISS in the prediction ability of death $[8,10,11]$, the SRR underestimates the survival rate of patients with a single injury and overestimates survival rate of patients with multiple injuries. Therefore, ICISS is inaccurate for the prediction of mortality (survival).

TMPM-ICD9 [9] derived an empirical severity value for each ICD-9-CM code that is called the modelaveraged regression coefficient (MARC) which is similar to TMPM [4]. Then, calculating the TMPM-ICD9 value according to MARC values by using a special formula. The TMPM-ICD9 is better than the ICISS as a predictor of mortality $[7,9]$. Researchers concluded that the TMPM-ICD9 outperforms the ISS and NISS in mortality prediction [7, 12]. TMPM-ICD9 is statistically rigorous, but it is not accurate enough in mathematics. There is a tendency to overestimate the severity of the injury [12].

We propose a new ICD-10-CM injury model which replaces the sole regression-based approach. Then we compare the performance of injury mortality prediction (IMP-ICDX), a new mortality prediction model based on these empiric injury severities, with ISS and single worst injury (SWI) models. Our objective was that the IMP-ICDX would provide a more accurate prediction of mortality than other existing scoring systems.

\section{Methods}

\section{Data source}

The patients came from the National Trauma Data Bank (NTDB) hospitalized between 2015 and 2016. Available information included patient demographics, ICD-10-CM diagnostic and injury codes (national clinical revision in American), mechanism of injury (according to ICD-10CM E-codes), ISS (version 2005), in-hospital mortality, Glasgow Coma Score (GCS), and encrypted hospital identifiers. This dataset consisted of 967,978 patients with 1 or more ICD-10-CM injury codes and AIS codes. Patients with non-traumatic diagnoses (e.g., drowning, poisoning, and suffocation) or burns $(47,184)$, missing or invalid data (data missing on length of hospital stay, age, gender, or outcome) $(26,177)$, missing cause of trauma (8938), or age younger than 1 year (3900) and older than 89 years $(60,917)$ were excluded from our analysis. The reason is that patients over the age of 89 were a separate age category in the NDTB and were assigned the value of -99 for their age. Patients who transferred to another facility $(37,014)$ or were dead on arrival to the hospital $(10,388)$ were also excluded. Some patients were excluded from the analysis because they have more than 1 exclusion criteria. ICD-10-CM E-codes were mapped to 1 of the 6 injury mechanisms by an experienced trauma surgeon: fall, motor vehicle crash, violence, gunshot wound, stab wound, and blunt injury. The final dataset included 794,098 patients admitted to 738 trauma centers. The details for recruitment are shown in Fig. 1.

\section{Overview of IMP-ICDX development}

In this research, $60 \%$ of the total dataset is used to evaluate trauma mortality rate (TMR) of different ICD-10-CM codes. The TMR values are calculated in Additional file 1. Based on TMR, number of body region (NBR), and body region (BR), we created three separate probit regression models by adding six additional variables: age, gender, GCS, ventilator, mechanism of injury, and hospital fixed effects to each of them. In the meantime, we applied optimal ratio of death probability for NBR and BR to modify the traumatic death probability (TDP) for TMR, to achieve an optimal value. The median of the three greatest (worst) TDP values was adopted as a weighted median death probability (WMDP) for each specific ICD-10-CM code (see Additional file 2).

Twenty percent of the dataset (IMP-ICDX development dataset) is used to evaluate IMP-ICDX. We apply logistic regression model to calculate coefficient of IMPICDX (Table 4) and deduce specific formula for the IMP-ICDX (see Additional file 3). Twenty percent of the dataset (internal validation dataset) is not used for the development of WMDP and IMP-ICDX to estimate the statistical performance of IMP-ICDX.

\section{Customization of trauma models}

This internal validation dataset enables us to test the performance of the ISS, SWI, and IMP-ICDX. ISS was computed according to Baker et al. [1]. A single worst injury (SWI) model was defined as the WMDP value for the worst injury (i.e., the greatest WMDP value). IMP-ICDX comprises the five most severe WMDP values according to injury severity; the product of the WMDP values for the two worst injuries is used as a variable and determines whether or not the two worst injuries are in the same BR and NBR 


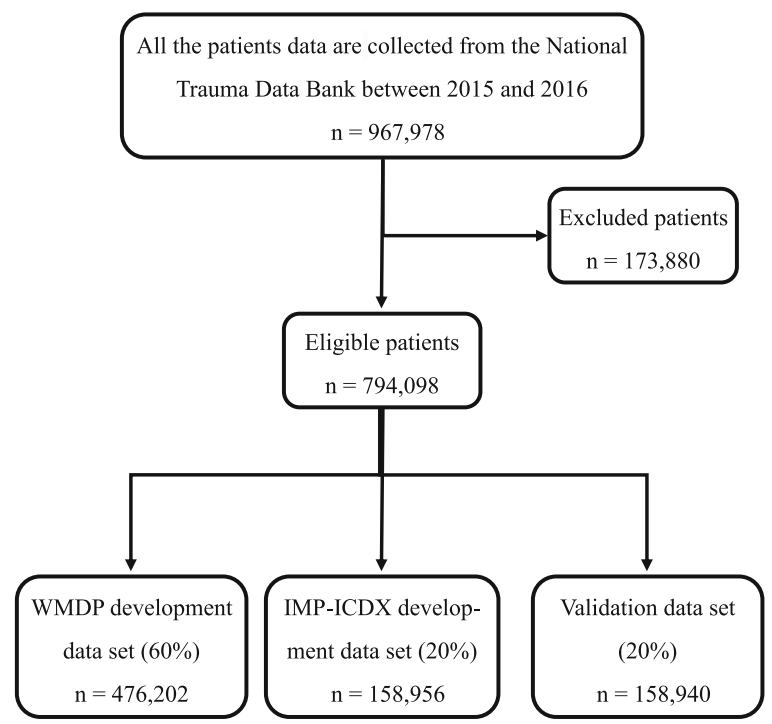

Fig. 1 Flowchart for data analyzed

(as $\ln (\mathrm{NBR})$ and $\mathrm{NBR}^{0.382}$, suggested by fractional polynomial analysis [13]) of each individual injury patient. The probability of death was calculated with the specific IMP-ICDX formula. At the same time, we then re-estimate all three models after adding age, gender, and injury mechanism to simple injury models, which only include the information on anatomic injury. Robust variance estimators [14] were applied because of the possible correlate outcomes of patients treated at the same trauma center.

\section{Statistical analysis}

This article assessed the statistical performance of all models using the area under the receiver operating characteristic (AUC) curve for discrimination, the HosmerLemeshow (HL) statistic for calibration, and the Akaike information criterion (AIC) for proximity to the true model. Non-parametric bootstrapping resampling algorithm with 1000 replications provided 95\% confidence intervals (CIs) for the AUC and HL statistic. A $P<0.05$ was considered statistically significant. All statistical analyses were performed using STATA/MP version 14.0 for Windows. This paper was exempt from review by the Institutional Review Board of Hangzhou Normal University, People's Republic of China.

\section{Results}

In this text, the total of the WMDP values is 8534 different ICD-10-CM coded injuries (see Additional file 4). These WMDP values range from 0.009 for a minor injury (ICD10-CM, S42.412A: "Displaced simple supracondylar fracture without intercondylar fracture of left humerus, initial encounter for closed fracture") to a value of 1.927 for a severe injury (ICD-10-CM, S06.5X7A: "Traumatic subdural hemorrhage with loss of consciousness of any duration with death due to brain injury, initial encounter"). Although trauma ICD-10-CM codes are not set by experts and cannot show information of traumatic severity, which are different from AIS codes, this research calculates the WMDP values of different ICD-10-CM codes and uses them to react to the degree of severity of trauma. We believe that these WMDP values are appropriate and in accordance with the actual situation of clinical, not our subjective assume.

Patient demographics are summarized in Table 1. The median age of our cohort was 49 years. Males accounted for $61.3 \%$, and $66.4 \%$ was non-Hispanic White. The majority of patients in this text were fall (44.4\%) and motor vehicle collisions (35.8\%). The overall mortality rate for the patients was $2.41 \%$.

The statistical performance of all models is shown in Tables 2 and 3. The IMP-ICDX displays significantly better discrimination, calibration, or AIC statistic compared with both the ISS and SWI models. Figure 2 graphically displays the superior calibration of IMP-ICDX. The ISS values were distributed to the right of the dotted reference line. The IMP-ICDX values were uniformly distributed much closer to the dotted reference line. The IMP-ICDX coefficients are shown in Table 4.

\section{Discussion}

The probability of death from patient trauma depends on many factors. The most important condition is the 
Table 1 Patient demographics

\begin{tabular}{ll}
\hline Patient characteristics & No. of patients (\%) \\
\hline Demographic variables & \\
Age, median (IQR) & $49(26-69)$ \\
Gender (male) & $486,930(61.3)$ \\
Mechanism of injury & \\
Fall & $352,479(44.4)$ \\
Motor vehicle accident & $284,329(35.8)$ \\
Violence & $59,906(7.6)$ \\
Gunshot & $35,883(4.5)$ \\
Stab & $35,234(4.4)$ \\
Blunt & $26,267(3.3)$ \\
Dead & $19,145(2.41)$ \\
\hline
\end{tabular}

$I Q R$ interquartile range

*Violence means to strike or against

patient's trauma severity. With the progress of medical science and the improvement of the treatment level, the trauma mortality has decreased obviously. Most of the existing trauma scores are difficult to distinguish real severity of all trauma patients, and even if there are trauma patients with similar severity, the results of treatment in different hospitals are also significantly differences [15]. This research also has similar results. For any individual patients, the likelihood of death is always accompanied by the whole course of treatment.

At present, there are many trauma score methods. For instance, ISS, NISS, and TISS are rapid evaluation methods while TMPM and IMP are retrospective evaluation methods, and they are all based on AIS codes. These methods have been widely used in clinical practice. They require that all patients have their injuries described in the AIS lexicon. Otherwise, they cannot be used to calculate, which limits their application. The ICISS and IMPMICD9, which are based on ICD-9-CM code, have broken away from the AIS code and opened up a new way of scoring method. TMPM-ICD9 is better than ICISS in predicting death results $[7,9]$. The data used in this study was derived from ICD-10-CM instead of ICD-9-CM. The above scoring methods are not suitable. Though ICD-10$\mathrm{CM}$ encoding can be converted to ICD-9-CD code and AIS code can be generated, the result after conversion is bound to be biased. It is not in line with the original

Table 2 Model performance: anatomic injury models

\begin{tabular}{llll}
\hline Model description & AUC $(95 \% \mathrm{Cl})$ & $\mathrm{H}-\mathrm{L}$ stat $(95 \% \mathrm{Cl})$ & $\mathrm{AIC}$ \\
\hline ISS & $0.853(0.846-0.860)$ & $252(191-310)$ & 27,655 \\
Single worst injury & $0.886(0.881-0.892)$ & $92(53-128)$ & 23,289 \\
IMP-ICDX & $0.893(0.887-0.898)$ & $68(36-98)$ & 23,024 \\
\hline
\end{tabular}

The IMP-ICDX demonstrated the best discrimination, calibration, and AIC compared to the single worst injury and ISS models
Table 3 Model performance: anatomic injury models augmented with age, gender, and injury mechanism

\begin{tabular}{llll}
\hline Model description & AUC $(95 \% \mathrm{Cl})$ & H-L stat $(95 \% \mathrm{Cl})$ & AIC \\
\hline ISS & $0.903(0.899-0.908)$ & $152(106-202)$ & 25,916 \\
Single worst injury & $0.915(0.910-0.919)$ & $37.4(18.3-52)$ & 21,970 \\
IMP-ICDX & $0.919(0.914-0.923)$ & $26.5(11.2-41)$ & 21,660
\end{tabular}

Although every model will be changed by the addition of more predictors, IMP-ICDX still shows superior model compared to the SWI and ISS models

intention of this research. Therefore, it is sensible to compare IMP-ICDX with ISS in our study.

This text combines the large dataset of NTDB and the feasible scoring method to evaluate the results of the trauma. The NTDB has the world's largest and the most credible trauma dataset and contains trauma data of different trauma centers in different regions of the USA. It includes information that offers us with research.

In this TMR development dataset, when the actual mortality rate of specific ICD-10-CM code is 0 , the TMR value is based on the death trend of the National Vital Statistics Reports in the United States in 2015 [16]. It is set as the median of the possible mortality rate (PMR_M) ( see Additional file 1) because the data is not normally distributed. There are 105 (only contains 370 patients) single or multiple injuries with $100 \%$ mortality, but these single or multiple injuries each has 80 or fewer cases, and there is only 1 case when the majority of code pairs have $100 \%$ mortality rate. This paper assumed that there was additional one survivor. Then, we calculated the TMR value, and it seemed to decrease death cases. In fact, this modified approach is appropriate and more in conformity with clinical practice.

This study uses TMR, NBR, and BR to create three separate probit regression models respectively for the specific ICD-10-CM code on different individual patients. Meanwhile, we apply optimal ratio of death probability for NBR and BR to modify the TDP for TMR, in order to acquire optimal value. This is a combination of rigorous statistical regression models and mathematical properties to improve the prediction accuracy. As individual's contribution to the death depends mainly on the three most severe traumas such as ISS, NISS, and TISS agents that have been confirmed, on a specific ICD-10$\mathrm{CM}$ code using different individual patients, the three largest TDP weighted median as its final value (i.e., WMDP) (see Additional file 2).

This study, in IMP-ICDX, when only the death probability value of the most severe injury was used, the coefficient of the worst injury was about four times the coefficient of minor injuries (results not presented). The absolute value of IMP-ICDX and SWI only differs by 0.007 , as well as overlapping confidence intervals. What is more, they are still statistically significant $(P<0.01)$, indicating that IMP-ICDX is better than SWI at 


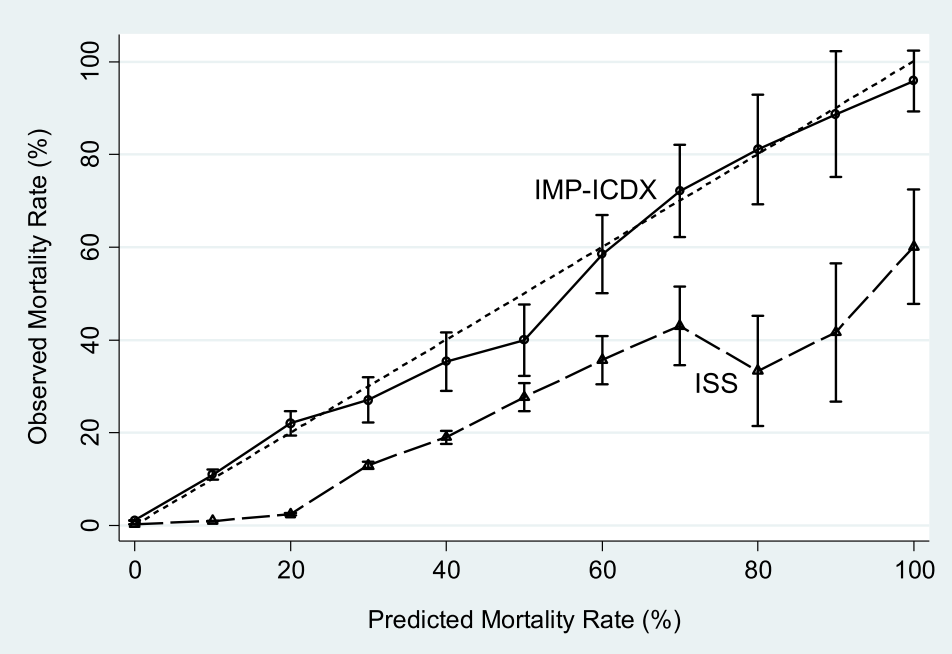

Fig. 2 Calibration curves for IMP-ICDX and ISS. The dotted reference lines represent perfect calibration (95\% binomial confidence intervals for IMP-ICDX and ISS models are based on the same validation dataset of 158,940 patients)

predicting traumatic death (Table 2). In a sense, SWI model to predict the death is also better [17]. Trauma surgeons usually describe a patient's clinical condition using the patient's one or two worst injuries. The TMPM-ICD9 holds that a patient's five worst injuries determine the possibility of mortality to a great extent [9], because in this dataset, only five coefficients of the most severe injuries in each patient were statistically significant (Table 4). Thus, IMP-ICDX is defined as the sum of the five worst WMDP values. The results greatly improve the accuracy of the predicted death, whether it is calibration, discrimination, or AIC statistics, far better than ISS (Table 2).
We found that the NBR and whether or not the use of mechanical ventilation in injured patients have intrinsic ability and useful parameters in predicting death due to trauma. They are better than patient's age or gender discrimination. As the existing evaluation methods (e.g., ICISS and TMPM-ICD9) were not involved, we added NBR and ventilator to improve IMP-ICDX trauma result prediction.

In general, additional information (such as respiratory rate, systolic blood pressure, and GCS) to anatomical injury score can always improve the predicted outcomes $[4,9,18]$. The fundamental IMP-ICDX is extremely attractive because only anatomical trauma information is

Table 4 IMP-ICDX regression coefficients

\begin{tabular}{|c|c|c|c|c|c|c|}
\hline Predictor & & Coefficient & Robust std. error & Z & $P>|Z|$ & $95 \% \mathrm{Cl}$ \\
\hline $\mathrm{WMDP}_{1}$ & $C_{1}$ & 4.9695 & 0.1198 & 41.49 & 0.000 & $4.7348-5.2043$ \\
\hline $\mathrm{WMDP}_{2}$ & $\mathrm{C}_{2}$ & 2.2315 & 0.2467 & 9.04 & 0.000 & $1.7479-2.7151$ \\
\hline $\mathrm{WMDP}_{3}$ & $C_{3}$ & 0.4111 & 0.1215 & 3.38 & 0.001 & $0.1730-0.6492$ \\
\hline $\mathrm{WMDP}_{4}$ & $\mathrm{C}_{4}$ & 0.3107 & 0.1418 & 2.19 & 0.028 & $0.0328-0.5885$ \\
\hline $\mathrm{WMDP}_{5}$ & $C_{5}$ & 0.3905 & 0.1318 & 2.96 & 0.003 & $0.1322-0.6488$ \\
\hline $\mathrm{WMDP}_{1} \times \mathrm{WMDP}_{2}$ & $\mathrm{C}_{6}$ & -0.7551 & 0.1428 & -5.29 & 0.000 & -1.0349 to -0.4753 \\
\hline Same region & $C_{7}$ & -0.2120 & 0.0488 & -4.34 & 0.000 & -0.3076 to -0.1163 \\
\hline In (NBR) & $\mathrm{C}_{8}$ & -2.4871 & 0.1942 & -12.80 & 0.000 & -2.8678 to -2.1064 \\
\hline $\mathrm{NBR}^{0.382}$ & $\mathrm{C}_{9}$ & 2.6147 & 0.2331 & 11.22 & 0.000 & $2.1578-3.0715$ \\
\hline Constant & $C_{0}$ & -10.7138 & 0.2630 & -40.73 & 0.000 & -11.2293 to -10.1983 \\
\hline
\end{tabular}

Coefficients for IMP-ICDX model were recalculated based on all 158,956 patients not used to calculate WMDP and IMP-ICDX values. WMDP ${ }_{1}$ indicates the worst injury (greatest WMDP value), $\mathrm{WMDP}_{2}$ the second worst injury, and so on. $\mathrm{WMDP}_{1} \times \mathrm{WMDP}_{2}$ is the product of the WMDP values for the 2 worst injuries. Same region is equal to 1 if the two worst injuries are in the same body region, 0 otherwise. NBR is the number of body regions for each injured patient. "In" indicates natural logarithm 
available. IMP-ICDX can also serve as a rich foundation in adding more sophisticated forecasting information to further enhance the accuracy of predicted results. The addition of the ventilator can enhance the AUC of the IMP-ICDX from 0.919 to 0.952 (no analysis). The IMPICDX had better discrimination and calibration than the ISS and the SWI models when we added age, gender, and injury mechanism (Table 3 ).

The goal of this research is to help people predict trauma death probability accurately according to the hospital diagnosis (ICD-10-CM coding), allocate medical resources rationally and effectively, guide clinical diagnosis and treatment, and ultimately improve the efficiency. This unique computing method can be applied to big data processing in other fields, which may lead to a revolutionary era of big data processing.

\section{Limitations}

The main limitation of this article is to inherit defects of the NTDB data. Although the data is bigger, it is not a population-based dataset. In addition, ICD-10$\mathrm{CM}$ coding may have differences because the data is derived from different trauma centers. At the same time, the ICD-10-CM code itself lacks the severity extent of the injury, which is different from the AIS code, and the prediction of the severity of traumatic death is not accurate; it is difficult to determine the injury severity of solid organs in particular, such as the liver, spleen, and kidney. ICD-10-CM codes have 8000 more variables and more than AIS codes, but they are still unable to make up for their defects. As there are too many encoding classifications, the number of single injury code of $60 \%$ data is 1988 and 689 codes are lost. If total data is used to calculate WMDP value or to increase the amount of data, the final AUC will be higher. ICD-10-CM-code-based IMP-ICDX outperforms ISS in predicting the death possibility. In this paper, the TMR value is used as a reference only; each TMR is required to be converted to WMDP by combining with the regression models and mathematical characteristics and then evaluating the probability of death of individual patients with different ICD-10-CM codes. Though the process of this calculation method is somewhat complicated, it can improve the ability to predict trauma death. A concurrent cohort study will likely have the same results, and those interested can test our results further.

\section{Conclusions}

In summary, IMP-ICDX is statistically significant compared to ISS, and its predictions of death, discrimination, and calibration are better than those of ISS. Therefore, in our opinion, IMP-ICDX could be a new feasible assessment method for trauma research.

\section{Supplementary information}

Supplementary information accompanies this paper at https://doi.org/10. 1186/s13017-019-0265-y

Additional file 1. The method of calculating TMR. (DOC 29 kb)

Additional file 2. Calculating WMDP values. (DOC $48 \mathrm{~kb}$ )

Additional file 3. Estimating IMP-ICDX. (DOC $22 \mathrm{~kb}$ )

Additional file 4. WMDP values of different ICD-10-CM injury codes. (XLS $1135 \mathrm{~kb})$

\section{Abbreviations}

AIC: Akaike information criterion; AIS: Abbreviated Injury Scale; AUC: Area under the receiver operating characteristic curve; BR: Body region;

Cl: Confidence interval; GCS: Glasgow Coma Score; H-L: Hosmer-Lemeshow; ICD-10-CM E-codes: International Classification of Diseases Tenth Revision

Clinical Modification External cause of injury codes; IMP: Injury mortality prediction; IMP-ICDX: Injury mortality prediction for ICD-10-CM;

IQR: Interquartile range; ISS: Injury severity score; In: Natural logarithm; MARC: Model-averaged regression coefficient; MMR: Multiple injuries mortality rate; NBR: Number of body region; NISS: New injury severity score; NTDB: National Trauma Data Bank; SMR: Single injury mortality rate; SRR: Survival risk ratio; SWI: Single worst injury; TDP: Trauma death probability; TISS: Tangent injury severity score; TMPM: Trauma mortality prediction model; TMPM-ICD9: Trauma mortality prediction model for ICD-9CM; TMR: Trauma mortality rate; WMDP: Weighted median death probability

\section{Acknowledgments}

The authors greatly appreciate the assistance from American College of Surgeons for providing data of the National Trauma Data Bank.

\section{Authors' contributions}

MW contributed to the study concept and design. MW, WQ, and YS contributed to the analysis and interpretation of data. All authors contributed to the critical revision of the manuscript for important intellectual content. MW contributed to the acquisition of data. WQ and MW contributed to the drafting of the manuscript. WF, $Y Z$, and $X L$ contributed to the literature search. All authors read and approved the final manuscript.

Funding

None.

Availability of data and materials

The data that support the findings of this study are available from NTDB databases of American College of Surgeons.

Ethics approval and consent to participate

Not applicable.

Consent for publication

Not applicable.

\section{Competing interests}

The authors declare that they have no competing interests.

\section{Author details}

${ }^{1}$ Department of Emergency Medicine, Affiliated Hospital of Hangzhou Normal University, 126 Wenzhou Road, Gongshu District, Hangzhou 310015, Zhejiang, People's Republic of China. 'Department of Neurosurgery, Affiliated Hospital of Hangzhou Normal University, Hangzhou 310015, Zhejiang, People's Republic of China. ${ }^{3}$ Department of Orthopedic, Affiliated Hospital of Hangzhou Normal University, Hangzhou 310015, Zhejiang, People's Republic of China. ${ }^{4}$ Department of Epidemiology and Health Statistics, School of Public Health, Zhejiang University, Hangzhou 310058, Zhejiang, People's Republic of China. 
Received: 22 July 2019 Accepted: 6 September 2019

Published online: 11 October 2019

\section{References}

1. Baker SP, O'Neill B, Haddon W, Long WB. The injury severity score: a method for describing patients with multiple injuries and evaluating emergency care. J Trauma. 1974;14:187-96.

2. Osler T, Baker SP, Long W. A modification of the injury severity score that both improves accuracy and simplifies scoring. J Trauma. 1997;43(6):922-6.

3. Wang MD, Qiu WS, Qiu F, Mo YN, Fan WH. Tangent function transformation of the Abbreviated Injury Scale improves accuracy and simplifies scoring. Arch Med Sci. 2015;11(1):130-6.

4. Osler T, Glance L, Buzas JS, Mukamel D, Wagner J, Dick A. A trauma mortality prediction model based on the anatomic injury scale. Ann Surg. 2008:247:1041-8.

5. Wang MD, Wu D, Qiu WS, Wang WM, Zeng YJ, Shen Y. An injury mortality prediction based on the anatomic injury scale. Medicine. 2017;96(35):e7945.

6. Keller WK, Dillihunt RC, Fenner HA, Jolley FL, Keeney AH, Weygandt PL, Hames LN. Rating the severity of tissue damage: I. the abbreviated injury scale. JAMA. 1971;215(2):277-80.

7. Cook A, Weddle J, Baker S, Hosmer D, Glance L, Friedman L, Osler T. A comparison of the injury severity score and the trauma mortality prediction model. J Trauma. 2013;76:47-53.

8. Osler T, Rutledge R, Deis J, Bedrick E. ICISS: an international classification of disease-9 based injury severity score. J Trauma. 1996;41:380-6.

9. Glance LG, Osler TM, Mukamel DB, Meredith W, Wagner J, Dick AW. TMPMICD9: a trauma mortality prediction model based on ICD-9-CM codes. Ann Surg. 2009;249:1032-9.

10. Meredith JW, Evans G, Kilgo PD, MacKenzie E, Osler T, McGwin G, et al. A comparison of the abilities of nine scoring algorithms in predicting mortality. J Trauma. 2002;53:621-9.

11. Hannan EL, Waller CH, Farrell LS, Cayten CG. A comparison among the abilities of various injury severity measures to predict mortality with and without accompanying physiologic information. J Trauma. 2005;58:244-51.

12. Haider AH, Villegas CV, Saleem T, Efron DT, Stevens KA, Oyetunji TA, et al. Should the ICD-9 Trauma Mortality Prediction Model become the new paradigm for benchmarking trauma outcomes? J Trauma Acute Care Surg. 2012;72(6):1695-701.

13. Sauerbrei $W$, Royston P. Building multivariable prognostic and diagnostic models: transformation of the predictors by using fractional polynomials. J R Stat Soc Ser A. 1999;162:71-94

14. White $\mathrm{H}$. A heteroskedasticity-consistent covariance matrix estimator and a direct test for heteroskedasticity. Econometrica. 1980;48:817-30.

15. Shafi S, Friese R, Gentilello L. Moving beyond personnel and process: a case for incorporating outcome measures in the trauma centre designation process. Arch Surg. 2008:143:115-9.

16. Murphy SL, Xu JQ, Kochanek KD, Curtin SC, Arias E. Deaths: Final data for 2015. National Vital Statistics Reports. Available at: http://www.cdc.gov/ nchs/data/nvsr/nvsr66/nvsr66 06.pdf. Accessed 27 Nov 2017.

17. Kilgo PD, Osler TM, Meredith $\bar{W}$. The worst injury predicts mortality outcome the best: rethinking the role of multiple injuries in trauma outcome scoring. J Trauma. 2003;55:599-606.

18. Boyd CR, Tolson MA, Copes WS. Evaluating trauma care: the TRISS method J Trauma. 1987;27:370-8.

\section{Publisher's Note}

Springer Nature remains neutral with regard to jurisdictional claims in published maps and institutional affiliations.

Ready to submit your research? Choose BMC and benefit from:

- fast, convenient online submission

- thorough peer review by experienced researchers in your field

- rapid publication on acceptance

- support for research data, including large and complex data types

- gold Open Access which fosters wider collaboration and increased citations

- maximum visibility for your research: over $100 \mathrm{M}$ website views per year

At $\mathrm{BMC}$, research is always in progress.

Learn more biomedcentral.com/submissions 